\title{
AutoDimer: a screening tool for primer-dimer and hairpin structures
}

\author{
Peter M. Vallone and John M. Butler \\ National Institute of Standards and Technology, Gaithersburg, MD, USA
}

BioTechniques 37:226-231 (August 2004)

The ability to select short DNA oligonucleotide sequences capable of binding solely to their intended target is of great importance in developing nucleic acid based detection technologies. Applications such as multiplex PCR rely on primers binding to unique regions in a genome. Competing side reactions with other primer pairs or template DNA decrease PCR efficiency. Freely available primer design software such as Primer3 screens for potential hairpin and primer-dimer interactions while selecting a single primer pair. The development of multiplex PCR assays (in the range of 5 to 20 loci) requires the screening of all primer pairs for potential cross-reactivity. However, a logistical problem results due to the number of total number of comparisons required. Comparing the primer set for a 10-plex assay (20 total primer sequences) results in 210 primer-primer combinations that must be screened. The ability to screen sets of candidate oligomers rapidly for potential cross-reactivity reduces overall assay development time. Here we report the application of a familiar sliding algorithm for comparing two strands of DNA in an overlapping fashion. The algorithm has been employed in a software package wherein the user can compare multiple sequences in a single computational run. After the screening is completed, a score is assigned to potential duplex interactions exceeding a user-defined threshold. Additional criteria of predicted melting temperature $\left(T_{m}\right)$ and free energy of melting $(\Delta G)$ are included for further ranking. Sodium counterion and total stand concentrations can be adjusted for the $T_{m}$ and $\Delta G$ calculations. The predicted interactions are saved in a text file for further evaluation.

\section{INTRODUCTION}

The ability to rapidly screen short DNA oligonucleotides for nonspecific interactions is essential for developing nucleic acid-based diagnostic tests. When designing primers or probes for multiplex applications such as PCR, primer extension, specific hybridization, events, etc., it is useful to have means to efficiently intercompare the primers/probes (1-9). The web-based primer selection program Primer3 currently selects PCR primers for generating a single PCR product (10). The selected forward and reverse primer sequences are screened for potential primer-dimer and intramolecular hairpin formation. Algorithms for screening complementarity between short DNA oligomers have been described (11). Various free and commercially available software packages for the selection of DNA probes and PCR primers employ variations on these basic algorithms for determining complementarity (12-16). The primary purpose of AutoDimer is to screen sets of preselected PCR primer pairs (ranging from 2 up to 1000 primer sequences) for potential cross-reactivity. The program performs primer-primer intercomparisons while evaluating interactions according to traditional Watson-Crick base pairing rules. The results can be visually inspected or saved to a text file. The information output consists of a visual component along with a score that represents the degree of interaction. Predictions of the transition melting temperature $\left(\mathrm{T}_{\mathrm{m}}\right)$ and free energy of melting $(\Delta \mathrm{G})$ are calculated for each of the potential primer-primer interactions.

\section{MATERIALS AND METHODS}

The algorithms for AutoDimer program were implemented in Visual Basic 6.0. The algorithm for determining interstrand complementarity is similar to that described earlier (11). Briefly, two sequences are incrementally overlapped, and the presence or absence of base pairing is evaluated and tabulated.
A schematic of the algorithm for the inter-comparison of two 20-nucleotide primers is depicted in Figure 1.

A variation upon this algorithm was employed for screening short intramolecular hairpin structures. An intramolecular hairpin can form when a single strand of DNA containing complimentary sequence regions binds to itself. The same single strand also has the potential to dimerize (intermolecularly), in which a duplex with a mismatched bulge will be present in the center of the duplex. The intermolecular homodimer will only be screened in the primer-dimer screening algorithm. DNA hairpins have a tendency to form at low salt and low strand concentrations in comparison to the corresponding homodimer. Traditionally, a hairpin consists of a stem (duplex region) and a single-stranded loop. The stem and loop designations are illustrated in Figure 2. The formation of a single-stranded loop is unfavorable and is typically associated with a positive free energy of formation. The stability of the duplex stem region must be high enough to overcome the destabilizing loop free energy. Studies on the effects of varying loop size and sequence have been reported $(17,18)$. Loops of 4 and 5 nucleotides have been found to be the least destabilizing. Based on this information, the hairpin screening algorithm allows for 4 and 5 base loops with a minimum of 2 base pairs in the stem. An example of the hairpin screening algorithm is depicted in Figure 2.

AutoDimer reads in an array of userdetermined sequences from a simple text file. Currently the program can read up to 1000 sequences from a single text file. The program has been packaged for installation and execution on a PC running Windows 98 and above.

A score value is determined by combining the number of Watson-Crick base pairs (+1) with mismatches (-1). Gaps and "N" nucleotides are not included in the score calculation, since the program is intended to screen short DNA oligomers. An example of a selfcomplementary sequence with a score of 7 is depicted in Figure 3. Estimates of $\mathrm{T}_{\mathrm{m}}\left(7.5^{\circ} \mathrm{C}\right)$ and $\Delta \mathrm{G}_{37^{\circ} \mathrm{C}}(-2.42 \mathrm{kcal} /$ mol) were calculated using nearestneighbor thermodynamic parameters. 
Table 1. Acceptable Input Formats for AutoDimer

\begin{tabular}{|ll|}
\hline Example $\left(\mathbf{5}^{\prime} \rightarrow \mathbf{3}^{\prime}\right)$ & Description \\
\hline >Sequence 1 & All lowercase \\
acacgtgtacatgtgtacacgtgt & \\
>Sequence 2 & Multiple spaces \\
gta cg tac gtac gta cgt ac gtac & \\
>Sequence 3 & Mixed upper/lowercase \\
GCGCGCGCcgcgcgcgcgcGCGCGCGC & \\
>Sequence 4 & Mixed upper/lowercase with spaces \\
atatat AT atatatatatatatatata & \\
\hline
\end{tabular}

The methods for performing these calculations are thoroughly described in the literature $(19,20)$.

\section{Input Sequence Format}

A simple text file is used for the sequence input. The " $>$ " character followed by a comment or label will designate the identity of the sequence that follows (similar to the FASTA type format). Currently the program will recognize upper and lower case A G C $\mathrm{T} U$ with or without spaces. Characters other than A G C T U are converted to $\mathrm{N}$ and treated as an unknown base (and as a mismatch in the base pairing rules). Each line of text is followed by a hard return. Additional hard returns are ignored. Examples of acceptable input formats are indicated in Table 1.

\section{User-Defined Parameters}

After the sequence list has been read into the program, the user can set the score threshold, sodium ion concentration $\left[\mathrm{Na}^{+}\right](\mathrm{mol} / \mathrm{L})$, total strand concentration $\left[\mathrm{C}_{\mathrm{T}}\right](\mu \mathrm{mol} / \mathrm{L})$, and the temperature at which $\Delta \mathrm{G}$ is calculated $\left(\Delta \mathrm{G}_{\text {temp }}\right)$. The score threshold determines which sequence interactions are stored to the output file. A sequence pair interaction that has a score of less than the threshold score is not saved or displayed in the program. Rerunning the program (with the same or different threshold score) results in a complete re-running of the algorithm (i.e., previous results are not retained unless manually saved). Note that varying $\left[\mathrm{Na}^{+}\right]$and/or $\mathrm{C}_{\mathrm{T}}$ will affect the estimated $\mathrm{T}_{\mathrm{m}}$ value $(19,20)$. Altering the $\mathrm{C}_{\mathrm{T}}$ will have not have an effect on the predicted $\Delta \mathrm{G}$. The temperature at which $\Delta \mathrm{G}$ is calculated can be varied depending on the intended use of the sequences. For example, one might set $\Delta \mathrm{G}_{\text {temp }}$ to a higher value than the $37^{\circ} \mathrm{C}$ default when screening PCR primers where the temperature may never fall below $50^{\circ} \mathrm{C}$. The program default values are $\left[\mathrm{Na}^{+}\right]=0.085 \mathrm{~mol} / \mathrm{L}, \mathrm{C}_{\mathrm{T}}=1.0$ $\mu \mathrm{mol} / \mathrm{L}$, and $\Delta \mathrm{G}_{\text {temp }}=37^{\circ} \mathrm{C}$.

\section{RESULTS AND DISCUSSION}

After loading the sequences into the program and varying any desired parameters, the user then selects either a primer-dimer or hairpin screen. A hairpin screen is usually not required if the primers were previously selected by another program. For example, default parameters in Primer3 allow for the screening of intramolecular hairpins before selecting an appropriate primer. After completion of the primer screening, the program displays interactions that meet the threshold criteria. A screenshot of the program after running a pair of primers is shown in Figure 4. For each interaction pair a score, estimated $\mathrm{T}_{\mathrm{m}}$, and $\Delta \mathrm{G}$ are provided. The program will indicate the number of hits and the number of total comparisons performed. For hairpins, the

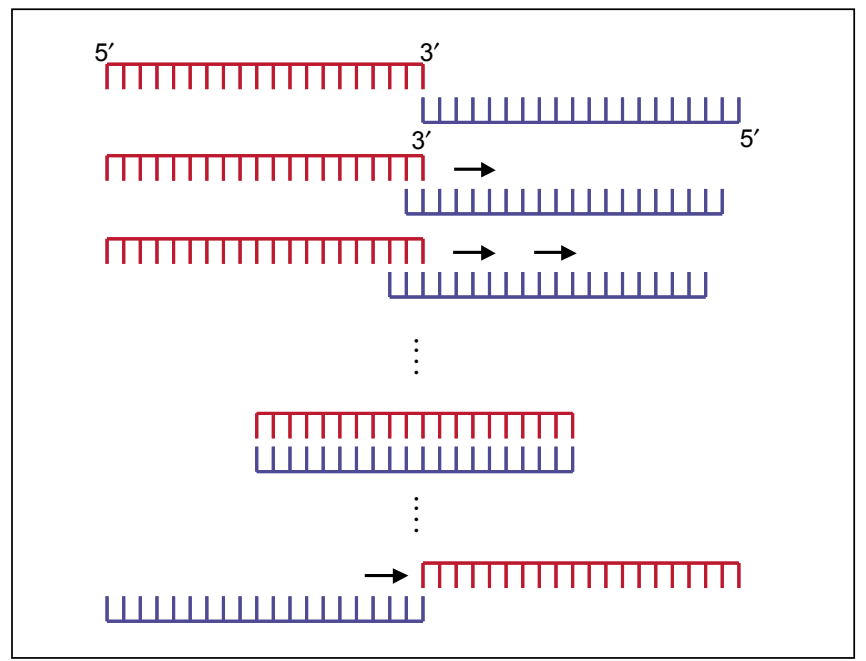

Figure 1. Basic sliding algorithm for screening secondary structures. Two duplexes are intercompared at each possible Watson-Crick base pairing state (excluding loops and gaps). In the example depicted above, 2 duplexes of length $\mathrm{M}$ and $\mathrm{N}$, where $\mathrm{M}=20$ and $\mathrm{N}=20$, results in 400 total comparisons. For a set of PCR primers, the number for duplex-duplex comparisons made is equal to $2 n^{2}+n$, where $n=$ number of primer pairs. For a 5 -plex ( 10 primer total), 55 primer-primer comparisons are made.

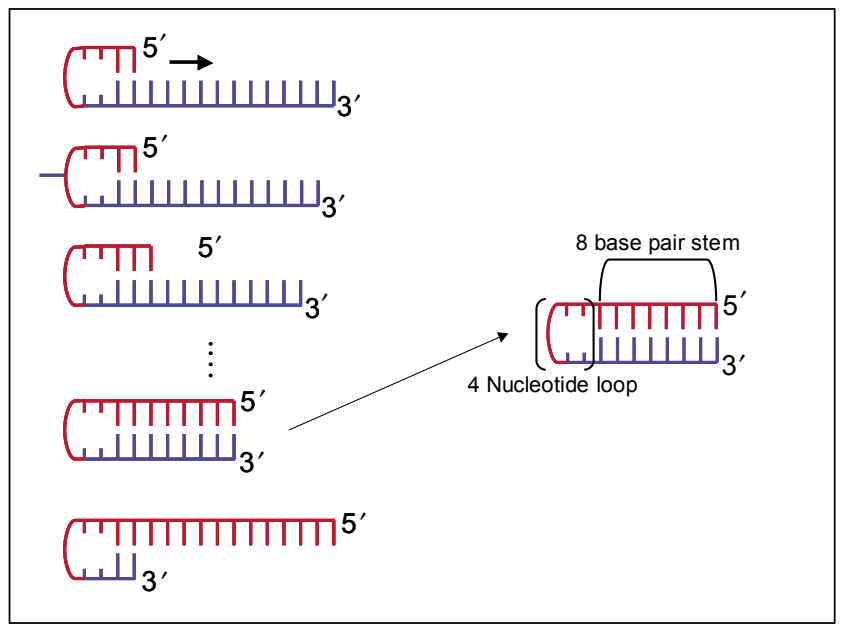

Figure 2. Sliding algorithm for screening intramolecular (hairpin) secondary structures. The algorithm allows for the presence of 4 and 5 base loops when screening. A minimum of a 2-base stem is allowed in a hairpin structure. In the depicted example, a single-stranded DNA oligomer is folded upon itself and screened for Watson-Crick base pairing. Screening the 20-nucleotide oligomer resulted in 116 base pair comparisons. The duplex stem and single-stranded loop regions of the hairpin are indicated to the right. 
number of comparisons is equal to the number of sequences screened, while for primer-dimers, the value is equal to $\left(n^{2}+n\right) / 2$, where $n$ is the total number of primers. The information can either be printed or stored to a text file for further evaluation. Table 2 indicates the computational run time and number of comparisons for screening a control set of 20-nucleotide length primers.

\section{Thermodynamic Values Versus Score Ranking}

It is necessary to provide information regarding the significance of the predicted interaction. The degree of importance will depend on the intended purpose of the sequence. For example, specific $3^{\prime}$-end interactions are especially significant for PCR and primer extension assays (21). In a chip-based hybridization-based assay, unwanted hybridization throughout the entire sequence could be significant. The level of multiplexing will also be of concern, since the greater the number of primers in solution results in the opportunity for decreased efficiency due to side hybridization reactions.

The score given for each interaction is a very basic criterion used to judge the degree of complementarity. The score reflects the general stability or tendency of the potential interaction to exist in solution. Data from our experiments suggests that a score threshold of 7 or 8 works well when designing multiplex PCR primers (22). A visual inspection of the interaction then allows the user to further decide whether the results are significant (based on 3 '-end binding, AT or GC content, context of mismatches,

\section{3'-GTCAGCTAGTAGCTAGCGCA-5' $|\mathbf{x x}||||| \mathbf{x}|||||\mathbf{x x}|$ 5'-ACGCGATCGATGATCGACTG-3'}

Figure 3. Results for screening a 20-nucleotide self-complementary sequence. The screening result for a 20-nucleotide sequence 5'-GTCAGCTAGTAGCTAGCGCA-3' is shown. The predicted interaction contains 12 Watson-Crick base pair matches and 5 mismatches. This results in a Score value of 7 (number of matches minus number of mismatches). Estimates for $\mathrm{T}_{\mathrm{m}}$ and $\Delta \mathrm{G}_{37^{\circ} \mathrm{C}}$ are $7.5^{\circ} \mathrm{C}$ and $-2.42 \mathrm{kcal} / \mathrm{mol}$, respectively. The sodium ion and $\mathrm{C}_{\mathrm{T}}$ were set to $0.085 \mathrm{M}$ and 1.0 $\mu \mathrm{M}$, respectively, for the $\mathrm{T}_{\mathrm{m}}$ and $\Delta \mathrm{G}_{37^{\circ} \mathrm{C}}$ calculations. $\mathrm{T}_{\mathrm{m}}$, melting temperature; $\Delta \mathrm{G}$, free energy of melting; $\mathrm{C}_{\mathrm{T}}$, total strand concentration.
Table 2. Run Time Parameters for a Control Set of Sequences

\begin{tabular}{|c|c|c|c|c|}
\hline \multirow{2}{*}{$\begin{array}{c}20 \text { Nucleotide } \\
\text { Primer Pairs } \\
\text { (N) }\end{array}$} & \multicolumn{2}{|c|}{ Duplex-Primer-Dimer } & \multicolumn{2}{|c|}{ Single Strand Hairpin } \\
\hline & Comparisons & $\begin{array}{c}\text { Run time }^{a} \\
\text { (s) }\end{array}$ & Comparisons & $\begin{array}{c}\text { Run time }^{a} \\
\text { (s) }\end{array}$ \\
\hline 1 & 3 & $<1$ & 2 & $<1$ \\
\hline 5 & 55 & 2 & 10 & 2 \\
\hline 10 & 210 & 6 & 20 & 2 \\
\hline 20 & 820 & 30 & 40 & 2 \\
\hline 50 & 5050 & $486(8 \mathrm{~min})$ & 100 & 3 \\
\hline 100 & 20,100 & $6960(1.9 \mathrm{~h})$ & 200 & 5 \\
\hline $408^{b}$ & 333,336 & $29,700(8.25 h)$ & 816 & 18 \\
\hline
\end{tabular}

etc.). By way of comparison, the webbased Primer 3 uses a score number of 8 as a default threshold for maximum selfcomplementarity (10).

A more quantitative way of describing the interaction is through a stability prediction based on predetermined thermodynamic parameters. Nearest neighbor thermodynamic parameters are evaluated from thermal melting experiments for a basis set of well-behaved (melting in a two-

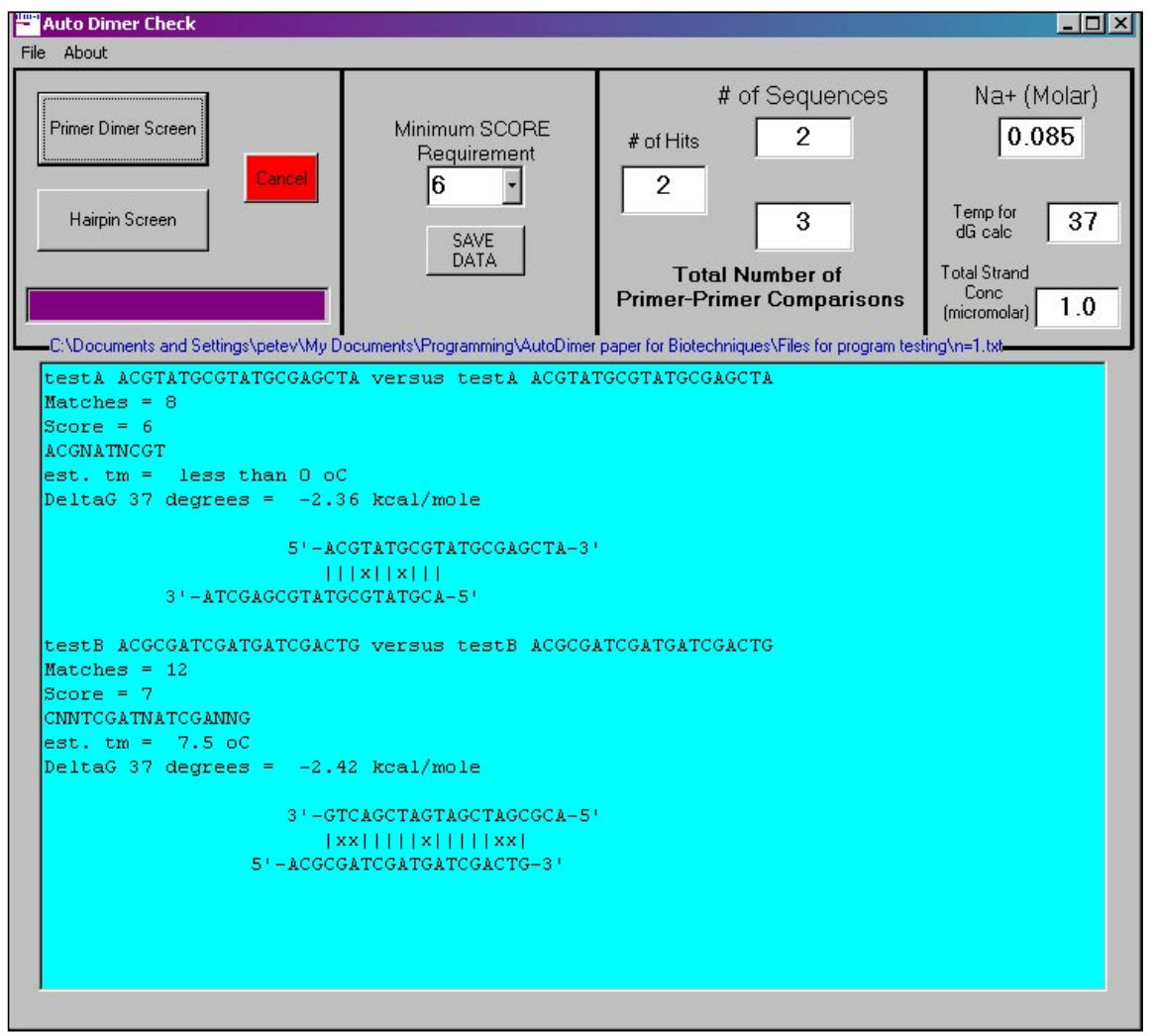

Figure 4. Screen shot of the AutoDimer software. A simple interface allows the user to open a sequence file. The program provides feedback on how many samples were read from the input file. The user can vary the score threshold, $\mathrm{Na}^{+}, \mathrm{C}_{\mathrm{T}}$, and $\Delta \mathrm{G}_{\text {temp }}$. Clicking on either the Primer Dimer Screen or Hairpin Screen buttons will start the appropriate algorithm, and results will be displayed in the window below. The screening results can be saved to a simple text file. The program also reports the total number of comparisons made, along with the number of hits as designated by the score threshold. $\Delta \mathrm{G}$, free energy of melting; $\mathrm{C}_{\mathrm{T}}$, total strand concentration. 
state manner) duplexes or minicircles (23-26). Control duplex or minicircle sequences are melted in a $\mathrm{Na}^{+}$solution commonly buffered with phosphate or cacodylate salts. The melting solutions rarely contain $\mathrm{Mg}^{2+}$, which can sometimes promote unwanted secondary structures. Mathematical analysis of the melting curves allow for the derivation of nearest-neighbor parameters that can be used to predict $\mathrm{T}_{\mathrm{m}}$ of the basis set duplexes as well as new sequences (23-26).

For the purpose of our program, we have included $\mathrm{T}_{\mathrm{m}}$ and $\Delta \mathrm{G}$ predictions for duplex stability based on the nearest-neighbor parameters of SantaLucia et al. (20). These parameters were derived from melting experiments involving short DNA oligomers. For short DNA duplexes, these parameters provide accurate predictions. A previously published empirical salt correction factor was used to adjust for differing ionic conditions (20). Single, tandem, and randomly spaced mismatched base pairing is common when evaluating the stability of duplex interactions. A complete set of thermodynamic parameters exists for the prediction of single base pair DNA mismatches (27-32). These parameters are intended for calculating the thermodynamic effect of a single base pair mismatch. Complexities exist due to multiple mismatches and mismatch context within a duplex $(33,34)$. Due to these issues, a conservative approach was implemented for dealing with mismatched base pairing. In the case of a single base pair mismatch, a penalty of $+438 \mathrm{cal} / \mathrm{mol}$ per mismatch was included in the $T_{m}$ and $\Delta G$ calculations regardless of the sequence of the mismatched base pair. This penalty is an estimate based on average destabilization due to mismatched base pairs evaluated in $1 \mathrm{M} \mathrm{Na}^{+}$(27-32). Specifically, the average cost of a mismatch is approximately $+438 \mathrm{cal} / \mathrm{mol}$ with an uncertainty of $\pm 509 \mathrm{cal} / \mathrm{mol}$. Therefore, predictions for $\mathrm{T}_{\mathrm{m}}$ and $\Delta \mathrm{G}$ values in this program should be regarded as a rough estimate for evaluating stability. For a more exact prediction of the effects of a single base mismatch in a short duplex, the exact nearest-neighbor parameters described by SantaLucia and coworkers can be used (27-32).

$\mathrm{T}_{\mathrm{m}}$ and $\Delta \mathrm{G}$ predictions for intramo- lecular DNA hairpins were calculated using methods described previously (35). The study involved melting experiments of short DNA hairpins with 4-nucleotide loops of varying sequence content. Based upon information from examining the stability of the short hairpins, an average loop destabilization of $\Delta \mathrm{G}_{37^{\circ} \mathrm{C}}=+$ $5.6 \mathrm{kcal} / \mathrm{mol}$ is assumed for 4 and 5 base loops regardless of sequence.

By employing a standard overlapping algorithm for the prediction of secondary hybridization events in combination with score and thermodynamic parameters, AutoDimer allows for rapid screening of short probe and primer oligonucleotides. The primary purpose of the software is to screen sets of previ- 
ously designed DNA probes and primers for potential cross-hybridization. AutoDimer allows rapid screening of short DNA oligonucleotide sequences for further review by a researcher based upon intended application. At this time, we are not aware of another standalone application for screening sets of sequences. The algorithm for screening is quite simple, but the power of the program lies in the ability to intercompare larger sets $(>2)$ of sequences. The resulting visual structures along with score or thermodynamic information provide useful feedback. This information can be used to evaluate primers that could be potentially paired off into a compatible multiplex set or, alternatively, used to determine if a particular interfering sequence should be redesigned.

The use of the AutoDimer screening tool has assisted in the development of an 11-plex PCR and primer extension assay for the detection of coding region single-nucleotide polymorphisms (SNPs) in the mitochondrial genome, a 20-plex PCR assay for typing Y-short tandem repeat (STR) loci, and various 5- and 6-plex PCR/primer extension assays used for evaluating Y-SNPs in U.S. sample groups (22,36-39). For each of these applications, PCR primers were first selected using Primer3 software. The primer pairs were placed into the appropriate format for the $\mathrm{Au}$ toDimer program and screened. If $\mathrm{Au}-$ toDimer screening results indicated significant complementarity, especially at the $3^{\prime}$ end of a primer (a stretch of 6 or more uninterrupted Watson-Crick base pairs), then the primer was redesigned. Typically, in a set of 20 primers, only one or two indicated any significant interactions. Due to the relatively computationally intensive nature of the algorithm we decided not employ the screening algorithm upstream in the primer selection process. The upstream approach involves screening a greater number of candidate primers for primer-dimer and hairpin interactions prior to being subjected to the traditional PCR primer selection criteria.

A strategy of comparing the primer pairs against the entire human genome [using the Basic Local Alignment Search Tool (BLAST) algorithm; http://www.ncbi.nlm.nih.gov/BLAST/] also helped to avoid secondary binding sites (40). This can be efficiently accomplished using the publicly available code on a local BLAST server for reasons of computational time.

As a larger test of the program, we screened 816 sequences (408 PCR primer pairs) used routinely for typing STR markers for genetic mapping purposes (the Set12 PCR primer sequences can be found at http://research. marshfieldclinic.org/genetics/sets/ combo.html). Typically, these are run as singleplex PCR. It would be advantageous to multiplex the compatible existing primer pairs. Setting a score threshold of 7 resulted in 1224 primerdimer interactions and no hairpin interactions. Over 333,336 primer comparisons were made in a little over $8 \mathrm{~h}$ of computational time. The 408 PCR primer sets and the results of primerdimer screening are posted at http:// www.cstl.nist.gov/biotech/strbase/ AutoDimerHomepage/Marshfield.htm.

AutoDimerv1 is available for free evaluation at the AutoDimer homepage http://www.cstl.nist.gov/biotech/ strbase/AutoDimerHomepage/AutoDimerProgramHomepage.htm. An installation package will set up the software to run on a PC running Window 98 or higher. A sample sequence input file and installation assistance is provided for the program.

\section{ACKNOWLEDGMENTS}

Certain commercial equipment, instruments, and materials are identified in order to specify experimental procedures as completely as possible. In no case does such identification imply a recommendation or endorsement by the National Institute of Standards and Technology (NIST) nor does it imply that any of the materials, instruments, or equipment identified are necessarily the best available for the purpose. The opinions and assertions contained herein are solely those of the authors and are not to be construed as official or as views of the U.S. Department of Commerce. The National Institute of Justice (NIJ) funded the work described here through an interagency agreement with the NIST Office of Law Enforcement Standards.

\section{COMPETING INTERESTS STATEMENT}

The authors declare no conflicting interests.

\section{REFERENCES}

1.Kaderali, L., A. Deshpande, J.P. Nolan, and P.S. White. 2003. Primer-design for multiplexed genotyping. Nucleic Acids Res. 31:1796-1802.

2.Kaderali, L. and A. Schliep. 2002. Selecting signature oligonucleotides to identify organisms using DNA arrays. Bioinformatics 18:1340-1349.

3.Kampke, T., M. Kieninger, and M. Mecklenburg. 2001. Efficient primer design algorithms. Bioinformatics 17:214-225.

4.Yuryev, A., J. Huang, M. Pohl, R. Patch, F. Watson, P. Bell, M. Donaldson, M.S. Phillips, and M.T. Boyce-Jacino. 2002. Predicting the success of primer extension genotyping assays using statistical modeling. Nucleic Acids Res. 30:e131.

5.Doi, K. and H. Imai. 1999. A greedy algorithm for minimizing the number of primers in multiple pcr experiments. Genome Inform. Ser. Workshop Genome Inform. 10:73-82.

6.Hyndman, D.L. and M. Mitsuhashi. 2003. PCR primer design. Methods Mol. Biol. 226:81-88.

7.Robertson, J.M. and J. Walsh-Weller. 1998. An introduction to PCR primer design and optimization of amplification reactions. Methods Mol. Biol. 98:121-154.

8.Rychlik, W. 1995. Selection of primers for polymerase chain reaction. Mol. Biotechnol. 3:129134.

9.Vieux, E.F., P.Y. Kwok, and R.D. Miller. 2002 Primer design for PCR and sequencing in highthroughput analysis of SNPs. BioTechniques (Suppl):28-32.

10.Rozen, S. and H. Skaletsky. 2000. Primer3 on the WWW for general users and for biologist programmers. Methods Mol. Biol. 132:365-386.

11.Rychlik, W. and R.E. Rhoads. 1989. A computer program for choosing optimal oligonucleotides for filter hybridization, sequencing and in vitro amplification of DNA. Nucleic Acids Res. 17:8543-8551.

12.Chen, S.H., C.Y. Lin, C.S. Cho, C.Z. Lo, and C.A. Hsiung. 2003. Primer Design Assistant (PDA): a web-based primer design tool. Nucleic Acids Res. 31:3751-3754

13.Chen, B.Y., H.W. Janes, and S. Chen. 2002. Computer programs for PCR primer design and analysis. Methods Mol. Biol. 192:19-29.

14.Gorelenkov, V., A. Antipov, S. Lejnine, N. Daraselia and A. Yuryev. 2001. Set of novel tools for PCR primer design. BioTechniques 31:13261330.

15.Haas, S., M. Vingron, A. Poustka, and S. Wiemann. 1998. Primer design for large scale sequencing. Nucleic Acids Res. 26:3006-3012.

16.Xu, D., G. Li, L. Wu, J. Zhou, and Y. Xu. 2002. PRIMEGENS: robust and efficient design of gene-specific probes for microarray analysis. Bioinformatics 18:1432-1437.

17.Groebe, D.R. and O.C. Uhlenbeck. 1988 Characterization of RNA hairpin loop stability. Nucleic Acids Res. 16:11725-11735.

18.Antao, V.P. and I. Tinoco, Jr. 1992. Thermo- 
dynamic parameters for loop formation in RNA and DNA hairpin tetraloops. Nucleic Acids Res. 20:819-824.

19.Owczarzy, R., P.M. Vallone, F.J. Gallo, T.M. Paner, M.J. Lane, and A.S. Benight. 1997. Predicting sequence-dependent melting stability of short duplex DNA oligomers. Biopolymers 44:217-239.

20.SantaLucia, Jr., J. 1998. A unified view of polymer, dumbbell, and oligonucleotide DNA nearest-neighbor thermodynamics. Proc. Natl. Acad. Sci. USA 95:1460-1465.

21.Ayyadevara, S., J.J. Thaden, and R.J. Shmookler Reis. 2000. Discrimination of primer 3'-nucleotide mismatch by Taq DNA polymerase during polymerase chain reaction. Anal. Biochem. 284:11-18.

22.Butler, J.M., C.M. Ruitberg, and P.M. Vallone. 2001. Capillary electrophoresis as a tool for optimization of multiplex PCR reactions. Fresenius J. Anal. Chem. 369:200-205

23.Breslauer, K.J., R. Frank, H. Blocker, and L.A. Marky. 1986. Predicting DNA duplex stability from the base sequence. Proc. Natl. Acad. Sci. USA 83:3746-3750.

24.Doktycz, M.J., R.F. Goldstein, T.M. Paner, F.J. Gallo, and A.S. Benight. 1992. Studies of DNA dumbbells. I. Melting curves of 17 DNA dumbbells with different duplex stem sequences linked by T4 endloops: evaluation of the nearest-neighbor stacking interactions in DNA. Biopolymers 32:849-864.

25.Sugimoto, N., S. Nakano, M. Yoneyama, and K. Honda. 1996. Improved thermodynamic parameters and helix initiation factor to predict stability of DNA duplexes. Nucleic Acids Res. 24:4501-4505.

26.SantaLucia, Jr., J., H.T. Allawi, and P.A. Seneviratne. 1996. Improved nearest-neighbor parameters for predicting DNA duplex stability. Biochemistry 35:3555-3562.

27.Allawi, H.T. and J. SantaLucia, Jr. 1997. Thermodynamics and NMR of internal GT mismatches in DNA. Biochemistry 36:10581-10594.

28.Allawi, H.T. and J. SantaLucia, Jr. 1998. Nearest-neighbor thermodynamics of internal $\mathrm{AC}$ mismatches in DNA: sequence dependence and $\mathrm{pH}$ effects. Biochemistry 37:9435-9444.

29.Allawi, H.T. and J. SantaLucia, Jr. 1998. NMR solution structure of a DNA dodecamer containing single GT mismatches. Nucleic Acids Res. 26:4925-4934.

30.Allawi, H.T. and J. SantaLucia, Jr. 1998. Thermodynamics of internal CT mismatches in DNA. Nucleic Acids Res. 26:2694-2701.

31.Allawi, H.T. and J. SantaLucia, Jr. 1998. Nearest neighbor thermodynamic parameters for internal GA mismatches in DNA. Biochemistry 37:2170-2179.

32.Peyret, N., P.A. Seneviratne, H.T. Allawi, and J. SantaLucia, Jr. 1999. Nearest-neighbor thermodynamics and NMR of DNA sequences with internal AA, CC, GG, and TT mismatches. Biochemistry 38:3468-3477.

33.Hall, T.S., P. Pancoska, P.V. Riccelli, K. Mandell, and A.S. Benight. 2001. Sequence context and thermodynamic stability of a single base pair mismatch in short deoxyoligonucleotide duplexes. J. Am. Chem. Soc. 123:11811-11812.

34.Riccelli, P.V., T.S. Hall, P. Pancoska, K.E. Mandell, and A.S. Benight. 2003. DNA sequence context and multiplex hybridization reactions: melting studies of heteromorphic duplex DNA complexes. J. Am. Chem. Soc. 125:141-150.

35.Vallone, P.M., T.M. Paner, J. Hilario, M.J. Lane, B.D. Faldasz, and A.S. Benight. 1999. Melting studies of short DNA hairpins: influence of loop sequence and adjoining base pair identity on hairpin thermodynamic stability. Biopolymers 50:425-442.

36.Butler, J.M., R. Schoske, P.M. Vallone, M.C. Kline, A.J. Redd, and M.F. Hammer. 2002. A novel multiplex for simultaneous amplification of $20 \mathrm{Y}$ chromosome STR markers. Forensic Sci. Int. 129:10-24.

37.Schoske, R., P.M. Vallone, C.M. Ruitberg, and J.M. Butler. 2003. Multiplex PCR design strategy used for the simultaneous amplification of $10 \mathrm{Y}$ chromosome short tandem repeat (STR) loci. Anal. Bioanal. Chem. 375:333-343.

38.Vallone, P.M., R.S. Just, M.D. Coble, J.M. Butler, and T.J. Parsons. 2004. A multiplex allele-specific primer extension assay for forensically informative SNPs distributed throughout the mitochondrial genome. Int. J. Legal Med. 118:147-157.

39.Vallone, P.M. and J.M. Butler. Y-SNP typing of U.S. African American and Caucasian samples using allele-specific hybridization and primer extension. J. Forensic Sci. (In press).

40.Altschul, S.F., W. Gish, W. Miller, E.W. Myers, and D.J. Lipman. 1990. Basic local alignment search tool. J. Mol. Biol. 215:403-410.

Received 10 March 2004; accepted 21 April 2004.

Address correspondence to Peter M. Vallone, National Institute of Standards and Technology, Biotechnology Division, 100 Bureau Drive, Mail Stop 8311, Gaithersburg, MD 20899, USA. e-mail: peter. vallone@nist.gov 